\title{
THE JUNE 2008 FLARE OF MARKARIAN 421 FROM OPTICAL TO TeV ENERGIES
}

I. Donnarumma ${ }^{1}$, V. Vittorini ${ }^{1,2}$, S. Vercellone ${ }^{3}$, E. Del Monte $^{1}$, M. Feroci ${ }^{1}$, F. D’Ammando ${ }^{1,2}$, L. Pacciani ${ }^{1}$, A. W. Chen $^{3,4}$, M. Tavani ${ }^{1,2}$, A. Bulgarellit ${ }^{5}$, A. Giuliani ${ }^{3}$, F. Longo ${ }^{6}$, G. Pucella ${ }^{1}$, A. Argan ${ }^{1}$, G. Barbiellini ${ }^{6}$, F. Boffellit ${ }^{7}$, P. Caraveo ${ }^{3}$, P. W. Cattaneo ${ }^{7}$, V. Cocco $^{1}$, E. Costa $^{1}$, G. De Paris ${ }^{1}$, G. Di Cocco ${ }^{5}$, Y. Evangelista ${ }^{1}$, M. Fiorini ${ }^{3}$, T. Froysland ${ }^{2,4}$, M. Frutti ${ }^{1}$, F. Fuschino ${ }^{5}$, M. Galli $^{8}$, F. Gianotti ${ }^{5}$, C. Labanti ${ }^{5}$, I. Lapshov ${ }^{1}$, F. Lazzarotto ${ }^{1}$, P. Lipari ${ }^{9}$, M. Marisaldi ${ }^{5}$, M. Mastropietro ${ }^{10}$, S. Mereghetti ${ }^{3}$, E. Morelli ${ }^{5}$, A. Morselli ${ }^{11}$, A. Pellizzoni ${ }^{3}$, F. Perotti ${ }^{3}$, P. Picozza ${ }^{11}$, G. Porrovecchio ${ }^{1}$, M. Prest ${ }^{12}$, M. Rapisarda ${ }^{13}$, A. Rappoldi ${ }^{7}$, A. Rubini ${ }^{1}$, P. Soffitta ${ }^{1}$, M. Trifoglio ${ }^{5}$, A. Trois ${ }^{1}$, E. Vallazza ${ }^{6}$, A. Zambra ${ }^{3}$, D. Zanello ${ }^{9}$, C. Pittori ${ }^{14}$, P. Santolamazza ${ }^{14}$, F. Verrecchia ${ }^{14}$, P. Giommi ${ }^{14}$, S. Colafrancesco ${ }^{14}$, L. Salotti ${ }^{15}$ (The AGile Team), M. Villata ${ }^{16}$, C. M. Raiteri ${ }^{16}$, W. P. Chen ${ }^{17}$, N. V. Efimova ${ }^{18}$, B. Jordan ${ }^{19}$, T. S. Konstantinova ${ }^{18}$, E. Koptelova ${ }^{17}$, O. M. Kurtanidze ${ }^{20,21,22}$, V. M. Larionov ${ }^{18,23}$, J. A. Ros ${ }^{24}$, A. C. Sadun ${ }^{25}$ (The GASP-WEBT Team), H. Anderhub ${ }^{26}$, L. A. Antonelli ${ }^{27}$, P. Antoranz ${ }^{28}$, M. Backes ${ }^{29}$, C. BaiXeras $^{30}$, S. Balestra ${ }^{28}$, J. A. Barrio ${ }^{28}$, H. BartKo ${ }^{31}$, D. Bastieri ${ }^{32}$, J. Becerra GonZález ${ }^{33}$, J. K. Becker ${ }^{29}$, W. BednareK ${ }^{34}$, K. Berger ${ }^{34}$, E. Bernardini ${ }^{35}$, A. Biland ${ }^{26}$, R. K. Bock ${ }^{31,32}$, G. Bonnoli ${ }^{36}$, P. Bordas ${ }^{37}$, D. Borla Tridon ${ }^{31}$,

V. Bosch-Ramon ${ }^{37}$, T. Bretz $^{38}$, I. Britvitch ${ }^{26}$, M. Camara ${ }^{28}$, E. Carmona ${ }^{31}$, A. Chillingarian ${ }^{40}$, S. CommichaU ${ }^{26}$, J. L. Contreras ${ }^{28}$, J. Cortina ${ }^{39}$, M. T. Costado ${ }^{33,41}$, S. Covino ${ }^{27}$, V. Curter ${ }^{29}$, F. Dazzi ${ }^{32}$, A. De Angelis ${ }^{42}$, E. De Cea del Pozo $^{43}$, R. de los Reyes ${ }^{28}$, B. De Lotto ${ }^{42}$, M. De Maria ${ }^{42}$, F. De Sabata ${ }^{42}$, C. Delgado Mendez ${ }^{33}$, A. Dominguez ${ }^{44}$,

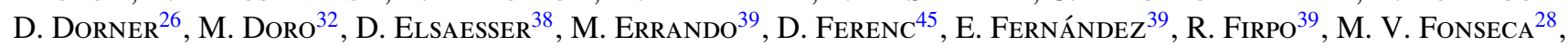

L. FonT ${ }^{30}$, N. Galante ${ }^{31}$, R. J. García LóPeZ ${ }^{33,41}$, M. GarCZarczyK ${ }^{39}$, M. Gaug ${ }^{33}$, F. GoebeL ${ }^{31,77}$, D. Hadasch ${ }^{30}$,

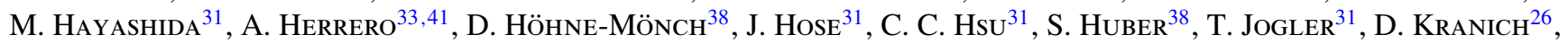
A. La Barbera ${ }^{27}$, A. Laille ${ }^{45}$, E. Leonardo ${ }^{36}$, E. LindFors ${ }^{46}$, S. Lombardi $^{32}$, M. LóPez ${ }^{32}$, E. Lorenz ${ }^{26,31}$, P. Majumdar ${ }^{35}$,

G. Maneva $^{47}$, N. Mankuzhiyil ${ }^{42}$, K. Mannheim ${ }^{38}$, L. Maraschi ${ }^{27}$, M. Mariotti $^{32}$, M. Martínez ${ }^{39}$, D. Mazin ${ }^{39}$,

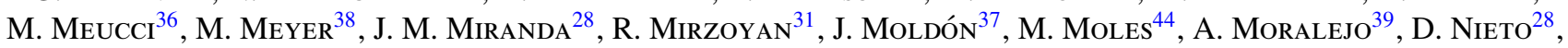
K. Nilsson ${ }^{46}$, J. Ninkovic ${ }^{31}$, I. Oya ${ }^{28}$, R. PaOletti ${ }^{36}$, J. M. Paredes ${ }^{37}$, M. Pasanen ${ }^{46}$, D. Pascol ${ }^{32}$, F. Pauss ${ }^{26}$, R. G. Pegna ${ }^{36}$, M. A. Perez-Torres ${ }^{44}$, M. Persic ${ }^{42,48}$, L. Peruzzo ${ }^{32}$, F. Prada $^{44}$, E. Prandini ${ }^{32}$, N. Puchades ${ }^{39}$, A. Raymers ${ }^{40}$, W. Rhode ${ }^{29}$, M. Ribó ${ }^{37}$, J. Rico ${ }^{49,39}$, M. Rissi ${ }^{26}$, A. Robert ${ }^{30}$, S. RÜGAMER ${ }^{38}$, A. SAGGion ${ }^{32}$, T. Y. SAito ${ }^{31}$, M. Salvati ${ }^{27}$,

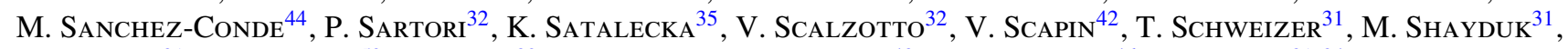
K. Shinozaki ${ }^{31}$, S. N. Shore ${ }^{50}$, N. Sidro ${ }^{39}$, A. Sierpowska-Bartosik ${ }^{43}$, A. Sillanpä̈ ${ }^{46}$, J. SitareK ${ }^{31,34}$, D. Sobczynska ${ }^{34}$, F. Spanier ${ }^{38}$, A. Stamerra ${ }^{36}$, L. S. Stark ${ }^{26}$, L. Takalo ${ }^{46}$, F. Tavecchio ${ }^{27}$, P. Temnikov ${ }^{47}$, D. Tescaro ${ }^{39}$, M. Teshima ${ }^{31}$,

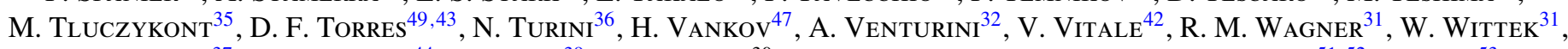

V. Zabalza ${ }^{37}$, F. Zandanel ${ }^{44}$, R. Zanin ${ }^{39}$, J. Zapatero ${ }^{30}$ (The MAGiC Collaboration), V. Acciari ${ }^{51,52}$, E. Aliu ${ }^{53}$,

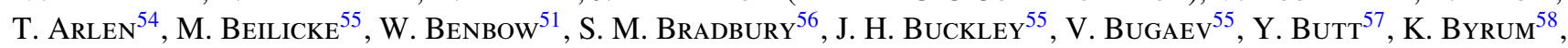
A. Cannon ${ }^{59}$, A. Cesarin ${ }^{60}$, Y. C. Chow $^{54}$, L. Ciupik ${ }^{61}$, P. Cogan ${ }^{62}$, P. Colin ${ }^{63}$, W. Cui ${ }^{64}$, M. K. Daniel ${ }^{56}$, R. Dickherber ${ }^{55}$, C. Duke ${ }^{65}$, T. Ergin $^{57}$, S. J. Fegan ${ }^{54}$, J. P. Finley ${ }^{64}$, G. Finnegan ${ }^{63}$, P. Fortin ${ }^{66}$, A. Furniss ${ }^{67}$, D. Gall ${ }^{64}$, G. H. Gillanders ${ }^{60}$, R. Guenette ${ }^{62}$, G. GyuK ${ }^{61}$, J. Grube ${ }^{56,59}$, D. Hanna ${ }^{62}$, J. Holder ${ }^{53}$, D. Horan ${ }^{58,76}$, C. M. Hui ${ }^{63}$, T. Brian HumenSKY ${ }^{68}$,

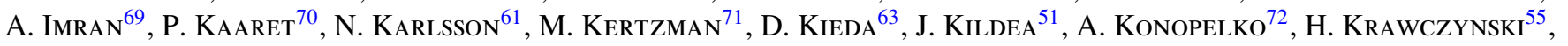

F. Krennrich ${ }^{69}$, M. J. Lang ${ }^{60}$, S. LeBohec ${ }^{63}$, G. Maier ${ }^{62}$, A. McCAnN ${ }^{62}$, M. McCutcheon ${ }^{62}$, A. Milovanovic ${ }^{56}$,

P. Moriarty ${ }^{52}$, T. Nagai ${ }^{69}$, R. A. Ong ${ }^{54}$, A. N. Otte ${ }^{67}$, D. Pandel ${ }^{70}$, J. S. Perkins ${ }^{53}$, A. Pichel ${ }^{73}$, M. Pohl ${ }^{69}$, K. Ragan ${ }^{64}$, L. C. Reyes ${ }^{74}$, P. T. Reynolds ${ }^{75}$, E. Roache ${ }^{51}$, H. J. Rose ${ }^{56}$, M. Schroedter ${ }^{69}$, G. H. Sembroski ${ }^{64}$, A. W. SMith ${ }^{58}$, D. STEELE ${ }^{61}$, S. P. Swordy ${ }^{68}$, M. Theiling ${ }^{51}$, J. A. Toner ${ }^{60}$, L. Valcarcel ${ }^{62}$, A. Varlotta ${ }^{64}$, S. P. Wakely ${ }^{68}$, J. E. Ward ${ }^{59}$, T. C. Weekes $^{51}$, A. Weinstein ${ }^{54}$, D. A. Williams ${ }^{67}$, S. Wissel ${ }^{68}$, M. Wood ${ }^{54}$, and B. Zitzer ${ }^{64}$ (The VERITAS Collaboration)

${ }^{1}$ INAF/IASF-Roma, I-00133 Roma, Italy; immacolata.donnarumma@iasf-roma.inaf.it

${ }^{2}$ Dipartimento di Fisica, Università Tor Vergata, I-00133 Roma, Italy

${ }^{3}$ INAF/IASF-Milano, I-20133 Milano, Italy

${ }^{4}$ CIFS-Torino, I-10133 Torino, Italy

${ }^{5}$ INAF/IASF-Bologna, I-40129 Bologna, Italy

${ }^{6}$ Dipartimento Fisica and INFN Trieste, I-34127 Trieste, Italy

${ }^{7}$ INFN-Pavia, I-27100 Pavia, Italy

${ }^{8}$ ENEA-Bologna, I-40129 Bologna, Italy

${ }^{9}$ INFN-Roma La Sapienza, I-00185 Roma, Italy ${ }^{10}$ CNR-IMIP, Roma, Italy

${ }^{11}$ INFN-Roma Tor Vergata, I-00133 Roma, Italy

12 Dipartimento di Fisica, Università Dell'Insubria, I-22100 Como, Italy

${ }^{13}$ ENEA Frascati, I-00044 Frascati (Roma), Italy

${ }^{14}$ ASI Science Data Center, I-00044 Frascati (Roma), Italy

${ }^{15}$ Agenzia Spaziale Italiana, I-00198 Roma, Italy

${ }^{16}$ INAF, Osservatorio Astronomico di Torino, Italy; villata@oato.inaf.it, raiteri@oato.inaf.it

${ }_{17}^{17}$ Institute of Astronomy, National Central University, Taiwan

${ }^{18}$ Astronomical Institute, St. Petersburg State University, Russia

${ }^{19}$ School of Cosmic Physics, Dublin Institute For Advanced Studies, Republic of Ireland

${ }^{20}$ Abastumani Astrophysical Observatory, Georgia

${ }^{21}$ Astrophysikalisches Institut Potsdam, Germany 
${ }^{22}$ Landessternwarte Heidelberg-Königstuhl, Germany

${ }^{23}$ Pulkovo Observatory, St. Petersburg, Russia

${ }^{24}$ Agrupació Astronòmica de Sabadell, Spain

${ }^{25}$ Department of Physics, University of Colorado at Denver, CO, USA

${ }^{26}$ ETH Zurich, CH-8093, Switzerland

${ }^{27}$ INAF National Institute for Astrophysics, I-00136 Rome, Italy

${ }^{28}$ Universidad Complutense, E-28040 Madrid, Spain

${ }^{29}$ Technische Universität Dortmund, D-44221 Dortmund, Germany

${ }^{30}$ Universitat Autònoma de Barcelona, E-08193 Bellaterra, Spain

${ }^{31}$ Max-Planck-Institut für Physik, D-80805 München, Germany; robert.wagner@mpp.mpg.de, cchsu@mpp.mpg.de

32 Università di Padova and INFN, I-35131 Padova, Italy

${ }^{33}$ Instituto de Astrofísica de Canarias, E-38200 La Laguna, Tenerife, Spain

${ }^{34}$ University of Łódź, PL-90236 Lodz, Poland

${ }^{35}$ Deutsches Elektronen-Synchrotron (DESY), D-15738 Zeuthen, Germany; satalk@ifh.de

${ }^{36}$ Università di Siena and INFN Pisa, I-53100 Siena Italy

${ }^{37}$ Universitat de Barcelona (ICC/IEEC), E-08028 Barcelona, Spain

${ }^{38}$ Universität Würzburg, D-97074 Würzburg, Germany

${ }^{39}$ IFAE, Edifici Cn., Campus UAB, E-08193 Bellaterra, Spain

${ }^{40}$ Yerevan Physics Institute, AM-375036 Yerevan, Armenia

${ }^{41}$ Departamento de Astrofisica, Universidad, E-38206 La Laguna, Tenerife, Spain

42 Università di Udine and INFN Trieste, I-33100 Udine Italy

${ }^{43}$ Institut de Cienciès de l'Espai (IEEC-CSIC), E-08193 Bellaterra, Spain

${ }^{44}$ Instituto de Astrofísica de Andalucia (CSIC), E-18080 Granada, Spain

${ }^{45}$ University of California, Davis, CA-95616-8677, USA

46 Tuorla Observatory, Turku University, FI-21500 Piikkiö, Finland

${ }^{47}$ Institute for Nuclear Research and Nuclear Energy, BG-1784 Sofia, Bulgaria

48 INAF/Osservatorio Astronomico and INFN, I-34143 Trieste, Italy

${ }^{49}$ ICREA, E-08010 Barcelona, Spain

${ }^{50}$ Università di Pisa, and INFN Pisa, I-56126 Pisa, Italy

${ }^{51}$ Fred Lawrence Whipple Observatory, Harvard-Smithsonian Center for Astrophysics, Amado, AZ 85645, USA

52 Department of Life and Physical Sciences, Galway-Mayo Institute of Technology, Dublin Road, Galway, Republic of Ireland

${ }^{53}$ Department of Physics and Astronomy and the Bartol Research Institute, University of Delaware, Newark, DE 19716, USA

${ }^{54}$ Department of Physics and Astronomy, University of California, Los Angeles, CA 90095, USA

${ }^{55}$ Department of Physics, Washington University, St. Louis, MO 63130, USA

${ }^{56}$ School of Physics and Astronomy, University of Leeds, Leeds LS2 9JT, UK; jeffrey.grube@ucd.ie

${ }^{57}$ Harvard-Smithsonian Center for Astrophysics, 60 Garden Street, Cambridge, MA 02138, USA

${ }^{58}$ Argonne National Laboratory, 9700 S. Cass Avenue, Argonne, IL 60439, USA

${ }^{59}$ School of Physics, University College Dublin, Belfield, Dublin 4, Republic of Ireland

${ }^{60}$ School of Physics, National University of Ireland, Galway, Republic of Ireland

${ }^{61}$ Astronomy Department, Adler Planetarium and Astronomy Museum, Chicago, IL 60605, USA

${ }^{62}$ Physics Department, McGill University, Montreal, QC H3A 2T8, Canada

${ }^{63}$ Physics Department, University of Utah, Salt Lake City, UT 84112, USA

${ }^{64}$ Department of Physics, Purdue University, West Lafayette, IN 47907, USA

${ }^{65}$ Department of Physics, Grinnell College, Grinnell, IA 50112-1690, USA

${ }^{66}$ Department of Physics and Astronomy, Barnard College, Columbia University, NY 10027, USA

${ }^{67}$ Santa Cruz Institute for Particle Physics and Department of Physics, University of California, Santa Cruz, CA 95064, USA

${ }^{68}$ Enrico Fermi Institute, University of Chicago, Chicago, IL 60637, USA

${ }^{69}$ Department of Physics and Astronomy, Iowa State University, Ames, IA 50011, USA

${ }^{70}$ Department of Physics and Astronomy, University of Iowa, Van Allen Hall, Iowa City, IA 52242, USA

${ }^{71}$ Department of Physics and Astronomy, DePauw University, Greencastle, IN 46135-0037, USA

72 Department of Physics, Pittsburg State University, 1701 South Broadway, Pittsburg, KS 66762, USA

${ }^{73}$ Casilla de Correo 67, Sucursal 28, (C1428ZAA) Ciudad Autònoma de Buenos Aires, Argentina

${ }^{74}$ Kavli Institute for Cosmological Physiscs, University of Chicago, Chicago, IL 60637, USA

${ }^{75}$ Department of Applied Physics and Instumentation, Cork Institute of Technology, Bishopstown, Cork, Republic of Ireland

${ }^{76}$ Laboratoire Leprince-Ringuet, Ecole Polytechnique, CNRS/IN2P3, F-91128, Palaiseau, France

Received 2008 October 15; accepted 2008 December 4; published 2008 December 29

\section{ABSTRACT}

We present optical, X-ray, high-energy $(\lesssim 30 \mathrm{GeV})$ and very high energy $(\gtrsim 100 \mathrm{GeV}$; VHE) observations of the high-frequency peaked blazar Mrk 421 taken between 2008 May 24 and June 23. A high-energy $\gamma$-ray signal was detected by AGILE with $\sqrt{T S}=4.5$ between June 9 and 15 , with $F(E>100 \mathrm{MeV})=42_{-12}^{+14} \times 10^{-8}$ photons $\mathrm{cm}^{-2} \mathrm{~s}^{-1}$. This flaring state is brighter than the average flux observed by EGRET by a factor of $\sim 3$, but still consistent with the highest EGRET flux. In hard X-rays (20-60 keV) SuperAGILE resolved a five-day flare (June 9-15) peaking at $\sim 55 \mathrm{mCrab}$. SuperAGILE, RXTE/ASM and Swift/BAT data show a correlated flaring structure between soft and hard X-rays. Hints of the same flaring behavior are also detected in the simultaneous optical data provided by the GASP-WEBT. A Swift/XRT observation near the flaring maximum revealed the highest 2-10 keV flux ever observed from this source, of $2.6 \times 10^{-9} \mathrm{erg} \mathrm{cm}^{-2} \mathrm{~s}^{-1}$ (i.e. $>100 \mathrm{mCrab}$ ). A peak synchrotron energy of $\sim 3 \mathrm{keV}$ was derived, higher than typical values of $\sim 0.5-1 \mathrm{keV}$. VHE observations with MAGIC and VERITAS between June 6 and 8 showed the flux peaking in a bright state, well correlated with the X-rays. This extraordinary set of simultaneous data, covering a 12-decade spectral range, allowed for a deep 
analysis of the spectral energy distribution as well as of correlated light curves. The $\gamma$-ray flare can be interpreted within the framework of the synchrotron self-Compton model in terms of a rapid acceleration of leptons in the jet.

Key words: BL Lacertae objects: individual (Mrk 421) - gamma rays: observations - galaxies: jets - radiation mechanisms: non-thermal - X-rays: galaxies

\section{INTRODUCTION}

Mrk 421 is a nearby blazar $(z=0.031)$ and one of the brightest BL Lac objects given its distance of $134.1 \mathrm{Mpc}$ $\left(H_{0}=71 \mathrm{~km} \mathrm{~s}^{-1} \mathrm{Mpc}^{-1}, \Omega_{m}=0.27, \Omega_{\lambda}=0.73\right)$. It was observed in $\gamma$-rays by EGRET (Lin et al. 1992), and it was the first extragalactic object detected at $E>500 \mathrm{GeV}$ (Punch et al. 1992). It belongs to the class of high-energy peaked BL Lac objects (HBLs) (Padovani \& Giommi 1995), i.e. radio-loud active galactic nuclei with high radio and optical polarization. Its spectral energy distribution (SED) is double humped with a first peak usually in the soft-to-medium X-ray range, and a second one at GeV-TeV energies (Sambruna et al. 1996; Fossati et al. 1998). The first hump is commonly interpreted as due to synchrotron radiation from high-energy electrons in a relativistic jet, while the origin of the second peak is still uncertain. In the leptonic scenario, it is interpreted as inverse Compton (IC) scattering of the synchrotron (synchrotron selfCompton, SSC) or external photons (external Compton, EC) by the same population of relativistic electrons. The observed correlated variability between $\mathrm{X}$-rays and $\mathrm{TeV} \gamma$-rays (Maraschi et al. 1999; Fossati et al. 2008, Wagner 2008) is well explained in the SSC framework (Ghisellini et al. 1998), whereas the EC scenario is unlikely to apply in HBLs, due to the low density of ambient photons. Alternatively, hadronic models invoke proton-initiated cascades and/or proton-synchrotron emission (Aharonian 2000, Mücke et al. 2003).

Leptonic and hadronic scenarios for HBLs predict different properties of the $\gamma$-ray emission in relation to emissions in other energy bands. Specifically, the hadronic models (as opposed to the SSC ones) predict a flatter slope of the $\sim 100 \mathrm{MeV}$ IC emission than that of the synchrotron emission in the optical, UV energy bands. $\gamma$-ray observations of flaring BL Lac objects and simultaneous multiwavelength data are, thus, the keys to investigating these two scenarios.

A hard X-ray flare of Mrk 421 was detected by SuperAGILE on 2008 June 10 (Costa et al. 2008). This detection was later followed by a detection in $\gamma$-rays (Pittori et al. 2008) by the $A G I L E / G R I D$ (gamma-ray imaging detector) and prompted a target of opportunity (ToO) observation by Swift/XRT, complementing the ongoing multifrequency observing campaign of Mrk 421 with WEBT (optical), MAGIC and VERITAS (TeV). We report on the observations and the analysis of these data, complemented by the publicly available data from $R X T E / A S M$ (2-12 keV) and Swift/BAT (15-50 keV), and discuss the spectral energy distribution of the source during this bright $\gamma$-ray flare.

\section{OBSERVATIONS AND RESULTS}

\subsection{AGILE Observations}

AGILE (Tavani et al. 2008) is a small mission of the Italian Space Agency for the exploration of the $\gamma$-ray sky, operating in a low Earth orbit since 2007 April 23. The AGILE composite payload allows for simultaneous observations in the energy ranges $30 \mathrm{MeV}-30 \mathrm{GeV}$ and $20-60 \mathrm{keV}$ over a very wide field of view (FOV) by means of GRID and the hard X-ray imager SuperAGILE, respectively. Mrk 421 was observed for five days, between 2008 June 9 17:02 UT and 2008 June 15 02:17 UT.

\subsubsection{Hard X-Ray Observations}

On 2008 June 10, SuperAGILE detected enhanced hard $\mathrm{X}$-ray emission from Mrk 421. The measured flux in 20-60 $\mathrm{keV}$ was found to be above $30 \mathrm{mCrab}$ ( $24 \mathrm{hr}$ average), almost an order of magnitude larger than its typical flux in quiescence. The source was at $\sim(0,20)$ degrees in the orthogonal SuperAGILE reference frame (Feroci et al. 2007), thus exposing an effective area $\sim 55 \%$ of the full on-axis value. In the following days, the flux increased up to about $55 \mathrm{mCrab}$. The five-day $20-60 \mathrm{keV}$ SuperAGILE light curve is shown in Figure 1(c). Using the publicly available light curves for this source from the BAT experiment $^{78}$ in the $15-50 \mathrm{keV}$ energy range (near the SuperAGILE bandpass), we calculated daily averages of the sparse observations. This allowed us to obtain good coverage also before and after the AGILE observation (black squares in Figure 1(c)), revealing that SuperAGILE indeed observed the maximum brightness of this hard X-ray flare. Both SuperAGILE and BAT count rates were converted to physical units by assuming a Crab-like spectrum.

SuperAGILE photon-by-photon data allow extraction of a time-averaged energy spectrum from the mask-convolved data. Given the lack of substantial spectral variability in the SuperAGILE/ASM hardness ratio (Figure 1(e)), we accumulated the average energy spectrum from the data of the last four days of the observations, when the source flux varied between 35 and $55 \mathrm{mCrab}$, for a total net source exposure of $\sim 140$ ks. Despite poor statistics, this four-point energy spectrum is able to reasonably constrain the photon index of a simple power law, $\Gamma=2.43_{-0.64}^{+0.69}\left(\chi_{\text {dof }}^{2}=0.8,2\right.$ dof $)$. The average flux is $F(20-60 \mathrm{keV})=(4.90 \pm 0.54) \times 10^{-10} \mathrm{erg} \mathrm{cm}^{-2} \mathrm{~s}^{-1}$ $\left((9.8 \pm 1.1) \times 10^{-3}\right.$ photons $\left.\mathrm{cm}^{-2} \mathrm{~s}^{-1}\right)$.

\subsubsection{Gamma-Ray Observations}

The GRID data were analyzed using the AGILE standard pipeline (Vercellone et al. 2008), with a bin size of $0.25 \times 0.25$. Only events flagged as $\gamma$-rays and not recorded while the satellite crossed the South Atlantic Anomaly were accepted. We also rejected all events with reconstructed direction within $10^{\circ}$ from the Earth limb, thus, reducing contamination from Earth's $\gamma$-ray albedo.

Mrk 421, observed $\sim 20^{\circ}$ off-axis with respect to the boresight, was not detected on daily time scales, implying a dailyaveraged flux smaller than $\sim 100 \times 10^{-8}$ photons $\mathrm{cm}^{-2} \mathrm{~s}^{-1}$, similar to what observed by EGRET (Hartman et al. 1999). A $4.5 \sigma$ significance in the range of $100 \mathrm{MeV}-10 \mathrm{GeV}$ resulted from an integration over the whole five-day period (net exposure $\sim 260 \mathrm{ks})$. The measured flux is $\left(42_{-12}^{+14}\right) \times 10^{-8}$ photons 


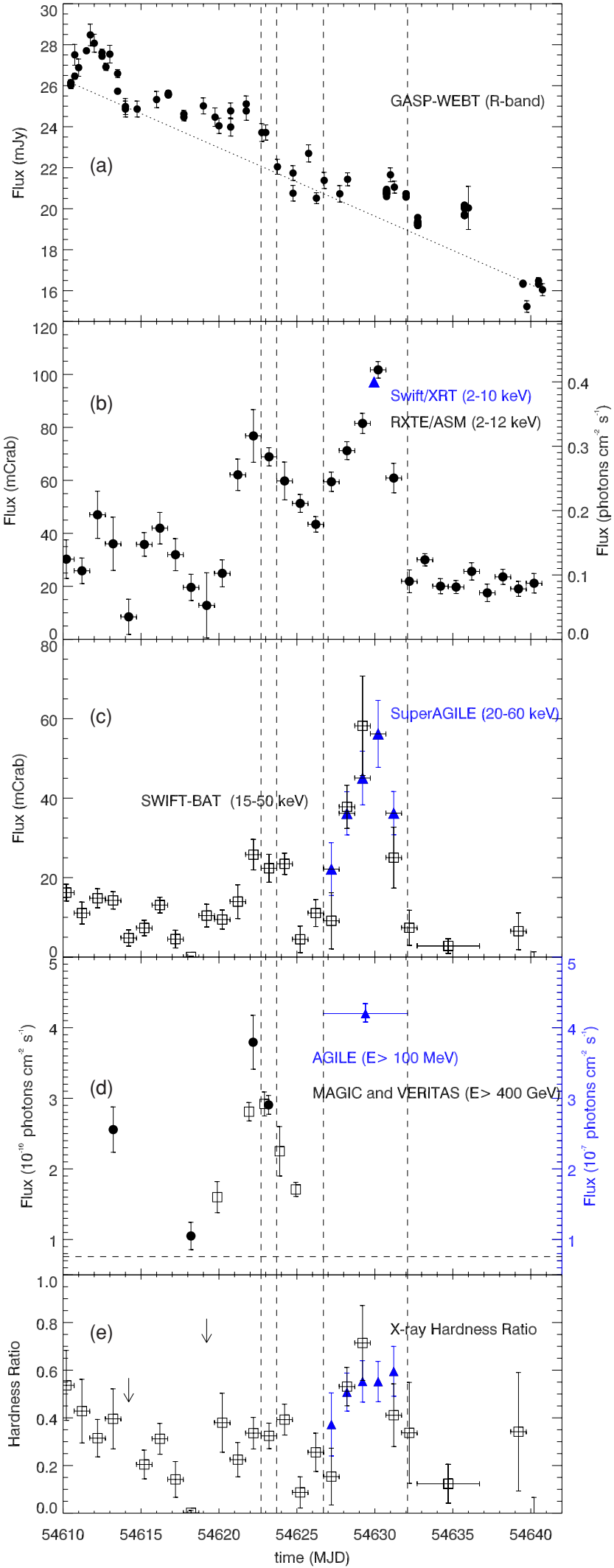

Figure 1. (a) $R$-band optical light curve from GASP-WEBT (May 24-June 23); (b) ASM (2-12 keV) light curve (bin size is one day) and XRT (2-10 keV) flux (blue triangle); (c) SuperAGILE (20-60 keV, blue triangles; 1 Crab = $0.2 \mathrm{ph} \mathrm{cm}^{-2} \mathrm{~s}^{-1}$ ) and BAT (15-50 keV, empty black squares; $1 \mathrm{Crab}=$ $0.29 \mathrm{ph} \mathrm{cm}^{-2} \mathrm{~s}^{-1}$ ); (d) MAGIC and VERITAS (>400 GeV, empty black squares and black circles, respectively), the Crab flux $>400 \mathrm{GeV}$ (horizontal dashed line), AGILE ( $>100 \mathrm{MeV}$, blue triangle); (e) hardness ratio computed using the SuperAGILE (or BAT) and ASM data for each day. The dashed vertical lines mark periods 1 and 2 (see the text for details). $\mathrm{cm}^{-2} \mathrm{~s}^{-1}$, about $\sim 3$ times higher than the average flux detected by EGRET $\left(\sim 13 \times 10^{-8}\right.$, Hartman et al. 1999) and $\sim 1.5$ times higher than, but still consistent with the highest flux $(27 \pm 7) \times 10^{-8}$ photons $\mathrm{cm}^{-2} \mathrm{~s}^{-1}$ observed during the Viewing Period 326. The short duration of the AGILE observation did not allow us to follow the possible decay of the $\gamma$-ray emission. Due to the relative faintness of the source and the short exposure, the GRID data do not allow us to extract a reliable energy spectrum.

\subsection{The Soft X-Ray Band}

\subsubsection{Swift XRT}

Following the SuperAGILE detection, on 2008 June 12 we triggered a ToO observation with the Swift/XRT that promptly observed the source for $5 \mathrm{ks}$ between 2008 June 12 19:33:20 UT (MJD 54629.8) and June 13 at 01:57:37 UT (MJD 54630.1).

The XRT data were processed using the standard procedure (xrtpipeline v0.12.0) developed at the ASI Science Data Center and distributed by the NASA/HEASARC. Observations were taken in Window Timing mode at a count rate of 120-150 counts $\mathrm{s}^{-1}$. We selected XRT grades 0-2 (Burrows et al. 2005) and extracted the WT data in a rectangular $40 \times$ 20 pixel region centered on the source. The background was also extracted within a box $(40 \times 20$ pixel $)$, far from background sources.

Due to calibration uncertainties, we restricted our spectral fit to the energy range $0.7-9.0 \mathrm{keV}$ and added a $3 \%$ systematics to the model (Cusumano et al. 2006). The XRT spectral data are well described by an absorbed log-parabolic model. A joint fit of the XRT and SuperAGILE spectral data using the four-day average spectrum described in Section 2.1.1. used the log-parabolic model with Galactic absorption $\left(N_{\mathrm{H}}^{\mathrm{Gal}}=1.61 \times\right.$ $10^{20} \mathrm{~cm}^{-2}$; Lockman \& Savage 1995), defined as

$$
F(E)=K E^{-a-b \log (E)} \text { photons } \mathrm{cm}^{-2} \mathrm{~s}^{-1},
$$

where $a$ is the photon index at $1 \mathrm{keV}$, and $b$ is the curvature parameter (Massaro et al. 2004; Massaro et al. 2008; Tramacere et al. 2007a, 2007b). This model usually describes adequately the featureless and curved spectrum in HBLs. The joint fit provides $a=1.65_{-0.02}^{+0.01}, b=$ $0.37_{-0.005}^{+0.01}\left(\chi_{\text {dof }}^{2}=1.4,763\right.$ dof $)$, which implies a peak energy $2.97_{-0.18}^{+0.22} \mathrm{keV}$, and predicts $F_{2-10 \mathrm{keV}}=2.56 \times$ $10^{-9} \mathrm{erg} \mathrm{cm}^{-2} \mathrm{~s}^{-1}$ (or 0.4 photons $\mathrm{cm}^{-2} \mathrm{~s}^{-1}$ ) and $F_{20-60 \mathrm{keV}}=$ $(5.7 \pm 0.6) \times 10^{-10} \mathrm{erg} \mathrm{cm}^{-2} \mathrm{~s}^{-1}\left((1.1 \pm 0.1) \times 10^{-2}\right.$ photons $\left.\mathrm{cm}^{-2} \mathrm{~s}^{-1}\right)$, comparable to the standalone SuperAGILE best fit.

\subsubsection{RXTE All Sky Monitor (ASM)}

Given the high flux observed by Swift/XRT, we retrieved the public light curves provided by the all sky monitor (ASM) ${ }^{79}$ to trace the evolution of the soft X-rays during the AGILE observation. Figure 1(b) shows the daily light curve of Mrk 421 in the energy range $2-12 \mathrm{keV}$, obtained by properly averaging the dwell-by-dwell data.

The emission at soft X-rays is well correlated with hard X-ray emission. The ASM data show that the XRT observation took place when the source was at its maximum emission at soft X-rays (MJD 54630). Comparing the relative intensity of the two flares in Figure 1, it appears that the second peak

\footnotetext{
79 http://xte.mit.edu/asmlc/ASM.html
} 
is significantly harder than the first one. This is also shown in Figure 1(e), where we computed the daily-averaged hardness ratio between hard (15-60 keV) and soft (2-12 keV) X-rays. The source appears to have undergone the hardest part of this double-humped flare just during the AGILE $\gamma$-ray detection.

\subsection{Observations in the VHE $\gamma$-Rays}

The four-telescope array VERITAS (Acciari et al. 2008) at the Fred Lawrence Whipple Observatory (Arizona) and the single-dish instrument MAGIC (Baixeras et al. 2004; Tescaro et al. 2007) at La Palma are imaging air Cherenkov telescopes covering an energy range from $\sim 100 \mathrm{GeV}$ to some tens of $\mathrm{TeV}$. The instruments have a typical energy resolution of $<20 \%$ (VERITAS) and 20-30\% (MAGIC), and event-by-event angular resolution of $<0$.14. Wobble-mode observations (Daum et al. 1997 ) at an 0.4 offset from the camera center were taken on five nights from 2008 June 3-8 with MAGIC at zenith angles (ZA) between $28^{\circ}$ and $48^{\circ}$ and on four nights (May 27, June 1, $5,6)$ with VERITAS (wobble offset: 0.5 ) at ZA between $32^{\circ}$ and $40^{\circ}$ during partial moonlight conditions. The total live time after applying quality selection is 2.95 and $1.17 \mathrm{hr}$ with MAGIC and VERITAS, respectively. The data were analyzed using the MAGIC (Albert et al. 2008a) and VERITAS (Daniel et al. 2007; Acciari et al. 2008) standard calibration and analyses and image parameters (Hillas 1985). For VERITAS, the $\gamma$-ray direction and air shower impact parameter on the ground are reconstructed using the stereoscopic techniques in Hofmann et al. (1999) and Krawczynski et al. (2006). Any $\gamma$-ray excess is derived from the $\theta^{2}$ distribution, where $\theta$ represents the angular distance between the source position in the sky and the reconstructed arrival position of the air shower. For MAGIC, $\theta$ is estimated using the DISP method (Fomin et al. 1994). For VERITAS, $99.9 \%$ of the background of cosmic rays is rejected by using selection cuts on $\theta^{2}$, mean scaled width and length, and by using quality cuts of each event. The MAGIC analysis utilizes a random forest method (Albert et al. 2008b) to discriminate the dominating background of hadronic cosmic-ray events and for the energy estimation of the $\gamma$-ray events. The energy and effective area of each event are reconstructed from Monte Carlo simulations. The integral flux and energy spectrum of the source are then derived from the effective areas for nights with a significant detection. Two independent analyses of both the MAGIC and VERITAS data sets yielded consistent results. In total, a signal corresponding to a significance level of $44 \sigma$ (VERITAS) and $66 \sigma$ (MAGIC) is obtained by following Equation (17) of Li \& Ma (1983). The combined MAGIC-VERITAS data (Figure 1(d)) show a transient peaking near MJD 54622. The VERITAS energy spectrum for June 6 is provided. A power-law fit over the energy range $0.3-5 \mathrm{TeV}$ resulted in a $\chi_{\text {dof }}^{2}=0.7$ with a photon index $\Gamma=2.78 \pm 0.09$. In Figure 2, we show the intrinsic $\gamma$-ray spectrum at the source reconstructed by removing attenuation effects by the extragalactic background light (Hauser \& Dwek 2001) following the procedure of Raue \& Mazin $(2008)^{80}$. Fitting a power law to the intrinsic spectrum yields a photon index $\Gamma=2.59 \pm 0.08$, which is not significantly harder than the measured spectrum due to the relatively low redshift $z=0.031$.

\subsection{Optical and UV Observations}

Mrk 421 is one of the $28 \gamma$-ray-loud blazars that are regularly monitored by the GLAST-AGILE Support Program

\footnotetext{
80 http://www.desy.de/ mraue/ebl/
}

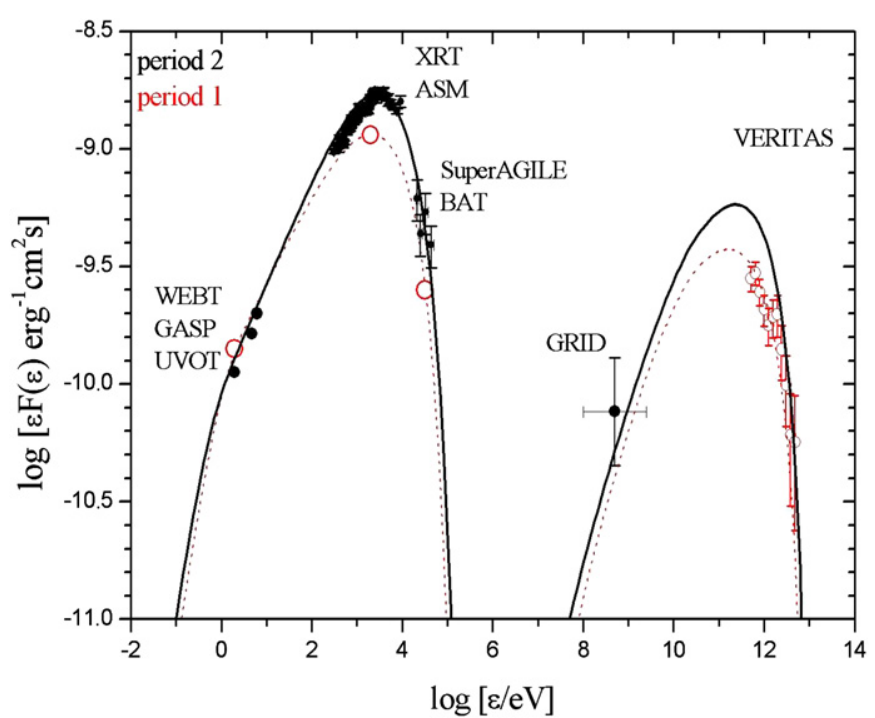

Figure 2. SEDs of Mrk 421 obtained by combining the GASP-WEBT, Swift/ UVOT, RXTE/ASM, XRT, SuperAGILE, BAT, GRID and VERITAS data in period 1 and period 2 (red empty circles and black filled circles, respectively). Both are one-zone SSC models (the red dashed line for period 1 and the black solid line for period 2).

(GASP; Villata et al. 2008) of the Whole Earth Blazar Telescope $(\text { WEBT })^{81}$. The GASP observations started in early 2007 November. The $R$-band data were calibrated according to the photometric sequence by Villata et al. (1998). A careful data analysis is warranted because the source flux is contaminated by the emission of the host galaxy and nearby objects. The flux contribution of these objects was subtracted according to Nilsson et al. (2007). Moreover, we corrected for a Galactic extinction of $A_{R}=0.042 \mathrm{mag}$. The resulting $R$-band light curve ${ }^{82}$ during the period considered in this Letter is shown in Figure 1(a).

During the Swift pointing on 2008 June 12-13, the UVOT (Poole et al. 2008) instrument observed Mrk 421 in the UVW1 and UVW2 photometric bands. The UVOTSOURCE tool is used to extract counts, correct for coincidence losses, apply background subtraction, and calculate the source flux. We applied a standard 5 arcsec radius source aperture, and a 20 arcsec background region. The source fluxes are dereddened using the interstellar extinction curve in Fitzpatrick (1999).

\section{DISCUSSION}

Mrk 421 showed very interesting broadband activity during the first half of 2008 June as derived from AGILE data combined with those of WEBT, Swift, MAGIC, and VERITAS. Using our multifrequency data, we were able to derive time-resolved SEDs (Figure 2). We distinguish two time periods: period 1: 2008 June 6, with the inclusion of optical, X-ray (XTE and BAT) and TeV data (VERITAS); and period 2: 2008 June 9-15, including optical, UV, X-rays (XRT and SuperAGILE) and gamma-ray data $(A G I L E)$. The source shows a very interesting time-variable broadband emission that appears to be in overall agreement with an SSC model. The optical, soft and hard X-ray bands strongly constrain the SED around the synchrotron peak, and its daily variability reveals the physical processes of Mrk 421. Possible correlated variability is shown in Figure 1 between the

\footnotetext{
81 http://www.oato.inaf.it/blazars/webt see, e.g. (Villata et al. 2007; Raiteri et al. 2007).

82 The optical data presented in this Letter are stored in the GASP-WEBT archive; for questions regarding their availability, please contact the WEBT President Massimo Villata at villata@oato.inaf.it.
} 
Table 1

SSC Model Parameters

\begin{tabular}{lcc}
\hline \hline Parameter & Period 1 & Period 2 \\
\hline$\gamma_{b}$ & $3.6 \cdot 10^{5}$ & $4.2 \cdot 10^{5}$ \\
$\gamma_{\max }$ & $1.3 \cdot 10^{6}$ & $1.3 \cdot 10^{6}$ \\
$p_{1}$ & 2.22 & 2.1 \\
$p_{2}$ & 4.5 & 5 \\
$B(\mathrm{G})$ & 0.1 & 0.1 \\
$K\left(\mathrm{~cm}^{-3}\right)$ & $4 \cdot 10^{-4}$ & $6 \cdot 10^{-4}$ \\
$\delta$ & 20 & 20 \\
$\theta\left[^{\circ}\right]$ & 2 & 2 \\
\hline
\end{tabular}

optical (an overall decreasing trend with superimposed spikes of emission), the X-rays (several emission peaks lasting few days), and the high-energy parts of the spectrum. Based on the physical constraints obtained for the synchrotron peak, we can model both the HE and VHE $\gamma$-ray emission. The data collection and broadband SED extend over 12 decades in energy. Taking advantage of the overlapping MAGIC and VERITAS observations, we present a combined VHE light curve using the current generation of northern imaging air Cherenkov systems.

We first model the synchrotron peak of emission using period 1 optical, soft and hard X-ray data. The short time variability (Figure 1) constrains the size of the emitting region to $R<c T \delta \sim 5 \times 10^{16}(\delta / 20) \mathrm{cm}$. Hence, we consider a one-zone SSC model (Tavecchio et al. 1998) based on a blob of comoving size $R=4 \times 10^{16} \mathrm{~cm}$, with a relativistic Doppler factor $\delta=20$ and characterized by nonthermal comoving electron energy distribution function described by a double power-law

$$
n_{e}(\gamma)=\frac{K \gamma_{b}^{-1}}{\left(\gamma / \gamma_{b}\right)^{p_{1}}+\left(\gamma / \gamma_{b}\right)^{p_{2}}}
$$

where the comoving Lorentz factor $(\gamma)$ varies in the range $\gamma_{\min }=4 \times 10^{3}<\gamma<\gamma_{\max }=1.3 \times 10^{6}$, the normalization (density) constant $K=4 \times 10^{-4} \mathrm{~cm}^{-3}$, and the break energy $\gamma_{b}=3.6 \times 10^{5}$ and with $p_{1}=2.22, p_{2}=4.5$, the low-energy and high-energy power-law indices, respectively (see Table 1). With these parameters, we found that the data for period 1 are best fitted with a comoving magnetic field $B=0.1 \mathrm{G}$.

Variability may be caused by several factors. We consider two cases: (A) hardening/softening of the electron energy distribution function caused by particle acceleration processes; (B) increase/decrease of the comoving particle density, as a consequence of additional particle injection/loss by shock processes.

We expect $\mathrm{TeV}$ variability to be comparable with the $\mathrm{X}$-ray one if case (A) applies: this is because the emission is in the Klein-Nishina regime. Alternatively, for case (B), we expect the $\mathrm{TeV}$ relative variability $(\Delta F / F)$ to be a factor of 2 greater than that of the X-ray flux variability.

Our AGILE, MAGIC, and VERITAS data appear to support case (A). We compare the SEDs for periods 1 and 2, to better assess the spectral evolution. In Figure 2, we show our optimized modeling of the time-resolved synchrotron peak and consequent SSC high-energy emission for period 1 as well as for 2008 June $12-13$ of period 2 . In the last case, the adopted model parameters are $p_{1}=2.1, p_{2}=5, \gamma_{b}=4.2 \times 10^{5}, K=6 \times 10^{-4} \mathrm{~cm}^{-3}$. Our model predicts an even larger TeV flux for period 2 (no TeV observations exist, however) than detected in period 1.

A detailed discussion of the complex optical versus X-ray variability of Mrk 421 as shown in Figure 1 will be presented elsewhere. We note here a few remarkable points. The optical light curve shows variations of the order of $10 \%$ on a timescale of a few days, superimposed on a long decay during the entire period. Individual soft and hard X-ray peaks result in increased fluxes by a factor of $\sim 2.5$ and $\sim 5$, respectively: no long-term decay appears. This different behavior of the X-ray radiation and the bulk of the optical emission may, interestingly, suggest more complex scenarios than (A) and (B): optical and X radiation come from two different jet regions, each one characterized by its own variability. A possible scenario is one in which the inner jet region would produce the X-rays, and it would be at least partially transparent to the optical radiation. In contrast, the outer region can only produce lower-frequency emission. The signature of the X-ray events visible in the optical light curve would come from the inner region and would be diluted by the optical radiation emitted from the outer region (see Villata \& Raiteri 1999 for the case of Mrk 501; Villata et al. 2004).

Interestingly, the $2-10 \mathrm{keV}$ flux measured by XRT on June $12-13, \sim 2.6 \times 10^{-9} \mathrm{erg} \mathrm{cm}^{-2} \mathrm{~s}^{-1}$, is higher than all previous observations $\left(<2 \times 10^{-9} \mathrm{erg} \mathrm{cm}^{-2} \mathrm{~s}^{-1}\right.$; Fossati et al. 2008; Lichti et al. 2008). A joint analysis of the XRT and SuperAGILE data, covering the range from 0.7 to $60 \mathrm{keV}$, provides a bestfit spectral model consistent with a log-parabolic shape, with parameters implying a peak energy $\sim 3 \mathrm{keV}$, in good agreement with the steeper positive correlation between the peak energy and the maximum of the SED found by Tramacere et al. (2007a) (see their Figure 3), although our value of the peak energy shows a significant shift with respect to typical values of $0.5-1 \mathrm{keV}$ for this source.

We conclude our analysis of the broadband variable emission from Mrk 421 by emphasizing that our multitelescope/ instrument data show a very interesting variability that provides support for an SSC model of the source. The $\gamma$-ray emission detected by AGILE during period 2 and the $\mathrm{TeV}$ emission detected during period 1 can be successfully modeled from the characteristics of the corresponding synchrotron peaks.

AGILE is a mission of ASI, with co-participation of INAF and INFN. This work was partially supported by ASI grants $\mathrm{I} / \mathrm{R} / 045 / 04, \mathrm{I} / 089 / 06 / 0, \mathrm{I} / 011 / 07 / 0$ and by the Italian Ministry of University and Research (PRIN 2005025417), (ASDC) I/024/05/1. The MAGIC collaboration thanks the Instituto de Astrofísica de Canarias for the excellent working conditions at the Observatorio del Roque de Los Muchachos in La Palma and support by the German BMBF and MPG, the Italian INFN and Spanish MCINN is acknowledged. This work was supported by ETH Research Grant TH 34/043, by the Polish MNiSzW Grant N N203 390834, the YIP of the Helmholtz Gemeinschaft, the grant of Georgian National Science Foundation GNSF/ST07/4180. E.K. acknowledges financial support from the NCS grant No. 96-2811-M-008-058. The VERITAS collaboration is supported by grants from the U.S. Department of Energy, the National Science Foundation, and the Smithsonian Institution, by NSERC in Canada, Science Foundation Ireland, and PPARC in the UK. We acknowledge the technical support staff at the FLWO. We also acknowledge the Swift Team for carrying out the ToO observation.

\section{REFERENCES}

Acciari, V. A., et al. (VERITAS Collaboration) 2008, ApJ, 679, 1427

Aharonian, F. A. 2000, NewA, 5, 377

Albert, et al. (MAGIC Collaboration) 2008a, ApJ, 674, 1037

Albert, et al. (MAGIC Collaboration) 2008b, Nucl. Instrum. Methods A, 588, 424 
Baixeras, C., et al. 2004, Nucl. Instrum. Methods A, 518, 188

Burrows, D. N., et al. 2005, Space Sci. Rev., 120, 165

Costa, E., et al. 2008, ATEL, 1574, 1

Cusumano, G. in Proc. Swift and GRBs: Unveiling the Relativistic Universe, June 5-9, 2006, Venice (Italy)

Daniel, M. K., et al. 2007, Proc. 30th ICRC Merida, Mexico, 283

Daum, A., et al. (HEGRA Collaboration) 1997, Astropart. Phys., 8, 1

Feroci, M., et al. 2007, Nucl. Instrum. Methods Phys. Res. A, 581, 728

Fitzpatrick, E. 1999, PASP, 111, 63

Fomin, V. P., et al. 1994, Astropart. Phys., 2, 137

Fossati, G., et al. 1998, MNRAS, 299, 433

Fossati, G., et al. 2008, ApJ, 677, 906

Ghisellini, G., et al. 1998, MNRAS, 301, 451

Hartman, R. C., et al. 1999, ApJS, 123, 79

Hauser, M. G., \& Dwek, E. 2001, ARA\&A, 39, 249

Hillas, A. M. 1985, in Proc. 19th ICRC, La Jolla, USA, 3, 445

Hofmann, W., et al. 1999, Astropart. Phys., 12, 135

Krawczynski, H., et al. 2006, Astropart. Phys., 25, 380

Lichti, G. G., et al. 2008, A\&A, 486, 721

Lin, Y. C., et al. 1992, ApJ, 401, 61

Lockman, F. J., \& Savage, B. D. 1995, ApJS, 97, 1

Li, T.-P., \& Ma, Y.-Q. 1983, ApJ, 272, 317

Maraschi, L., et al. 1999, ApJ, 526L, 81M

Massaro, E., et al. 2004, A\&A, 422, 103
Massaro, F., Tramacere, A., Cavaliere, A., Perri, M., \& Giommi, P. 2008, A\&A 478, 395

Mücke, A., et al. 2003, Astropart. Phys., 18, 593

Nilsson, K., et al. 2007, A\&A, 475, 199

Padovani, P., \& Giommi, P. 1995, ApJ, 444, 567

Pittori, C., et al. 2008, ATEL, 1583, 1

Poole, T. S., et al. 2008, MNRAS, 383, 627

Punch, M., et al. 1992, Nature, 358, 477

Raiteri, C. M., et al. 2007, A\&A, 473, 819

Raue, M., \& Mazin, D. 2008, Int. J. Mod. Phys. D, 17, 1515

Sambruna, R. M., Maraschi, L., \& Urry, C. 1996, ApJ, 463, 444

Tavani, et al. 2008, arXiv:0807.4254v1

Tavecchio, F., et al. 1998, ApJ, 509, 608

Tescaro, D., et al. (MAGIC Collaboration) 2007, in Proc. 30th ICRC, Merida, Mexico (arXiv:0709.1410)

Tramacere, A., et al. 2007a, A\&A, 466, 521T

Tramacere, A., et al. 2007b, A\&A, 467, $501 \mathrm{~T}$

Vercellone, S., et al. 2008, ApJ, 676, 13

Villata, M., \& Raiteri, C. M. 1999, A\&A, 347, 30

Villata, M., et al. 1998, A\&AS, 130, 305

Villata, M., et al. 2004, A\&A, 421, 103

Villata, M., et al. 2007, A\&A, 464, L5

Villata, M., et al. 2008, A\&A, 481, L79

Wagner, R. M. 2008, PoS (BLAZARS2008), 63, 013 (arXiv:0809.2483) 